\title{
Nuclear and Gamma-ray Production by Supernova Ejecta
}

\author{
Brian D. Fields, ${ }^{1,2}$ Michel Cassé,${ }^{3}$ Elisabeth Vangioni-Flam, ${ }^{1}$ and Ken'ichi Nomoto ${ }^{4}$ \\ ${ }^{1}$ Institut d'Astrophysique \\ 98 bis, boulevard Arago, Paris 75014, France \\ ${ }^{1}$ Department of Physics, University of Notre Dame \\ Notre Dame, IN 46556 USA \\ ${ }^{2}$ CE-Saclay, DSM/DAPNIA/Service d'Astrophysique \\ 91191 Gif sur Yvette cedex, France \\ ${ }^{3}$ Department of Astronomy and Research Center for the Early Universe \\ University of Tokyo, Tokyo, Japan
}

\begin{abstract}
The fastest ejecta of supernova explosions propagate as a precursor to the main supernova shock wave, and can be quite energetic. The spectrum of such fast ejecta is estimated based on recent analytic and numerical supernova models, and found to be a power law having a cutoff at an energy of order $10 \mathrm{MeV} /$ nucl, the precise value of which depends on the supernova mass and energetics. With cutoffs in this range there can be significant flux with energies above the thresholds for $\gamma$-ray and $\mathrm{Li}, \mathrm{Be}$, and B production. These nuclear interactions should inevitably accompany the passage of prompt supernova ejecta through the surrounding medium. The fast particle composition is that of the outermost layers of the progenitor; if the progenitor experienced significant loss of its envelope to a companion or by mass loss, then the composition is nonsolar, and in particular, metal-rich. Such a composition and spectrum of fast particles is required to explain the recent COMPTEL observations of $\gamma$-ray emission in Orion. Supernova ejecta from one progenitor having lost a large amount of mass are shown to quantitatively account for the Orion $\gamma$ rays, and to imply the presence of other, weaker lines and of a supernova explosion in Orion in the last $\lesssim 10^{4}$ years. Implications for this mechanism as a mode of nucleosynthesis, and as a potential supernova diagnostic, are discussed. Model dependences and uncertainties are noted, and the need is shown for accurate measurements of $\gamma$-ray and spallation production cross sections at energies near threshold.
\end{abstract}

Subject headings: acceleration of particles - gamma rays:theory - nuclear reactions, nucleosynthesis, abundances - supernovae:general 


\section{Introduction}

An exploding supernova is one of the most powerful engines in the Galaxy. Modern supernova models predict that the ejecta should have a distribution in velocity, with the outermost layers being ejected at great speed. Indeed, it was originally hoped (e.g., Colgate \& Johnson 1960) that these energetic particles might be the cosmic rays, and thus supernovae would be the direct agents of cosmic ray acceleration. This has been shown to be incorrect (e.g., Cassé \& Goret 1978; Meyer 1985a, 1985b), and instead it is now believed that the supernova blast leads indirectly to cosmic ray production via shock acceleration (as reviewed, e.g., in Blanford \& Eichler 1987, and Jones \& Ellison 1991).

Nevertheless, very fast ejecta are indeed created by supernova explosions. Indeed, some have energies above thresholds for nuclear interactions, in particular for $\gamma$-ray and $\mathrm{Li}, \mathrm{Be}$, \& $\mathrm{B}(\mathrm{LiBeB})$ production. These interactions should inevitably accompany the passage of fast ejecta through the medium surrounding the supernova. Given the presence of these interactions, the crucial question is whether they are numerous enough to be detectable. Indeed, if the flux of the fast particles is sufficiently high, the $\gamma$-rays could offer a new and unique window on the explosion, and the $\mathrm{LiBeB}$ could provide a record of such events, integrated over the history of the Galaxy.

In this paper we estimate the flux of the fast supernova ejecta and find that it is in fact sufficiently energetic and intense to lead to significant $\gamma$-ray production, and less significant LiBeB production. It is thus plausible that this mechanism is of great interest as a diagnostic of supernovae in general. More particularly, it is interesting in light of recent Orion $\gamma$-ray observations, which can be accounted for by prompt ejecta a single type Ic supernova (if it indeed arises from a CO core progenitor as recently suggested by Nomoto et al. 1994).

\section{Composition and spectrum of ener- getic massive star ejecta}

Consider a supernova exploding in some medium, e.g. the ISM or, in the case of Orion, a molecular cloud. The ejecta will interact with this medium, which may have been altered locally by the supernova progenitor. For example, if there was a strong wind, it will have cleared out a cavity in the local medium in which the local material is replaced by that of the wind. Moreover, a typical progenitor begins life as an O star whose UV radiation partially clears out the region near the star. In fact, it is only important for our purposes that the explosion be in some medium, and we will see that the particles we are interested in will travel much further than the lengthscale of local inhomogeneities.

After the explosion the bulk of the material will form a shock and pass through the usual phases (free-coasting, Sedov, etc.). But the precursors to the blast, the fastest particles ejected, are dilute and will not participate in the shock. They will instead propagate out and begin to lose energy predominantly through ionization losses, eventually to be stopped. These are the particles in which we are interested. Specifically, we want to calculate the intensity, spectrum, composition, and duration of the precursor nuclei.

Before making detailed calculations, let us fix the order of magnitude of the effect. Typical supernova velocity spectra have maximum velocities of the order of $40,000 \mathrm{~km} / \mathrm{s}$, i.e., with particle energies (per nucleon) up to $8 \mathrm{MeV} /$ nucl. Typical $\gamma$-ray thresholds (for reactions between an $\alpha$ particle and a heavy nucleus) are $\sim 2 \mathrm{MeV} /$ nucl, and spallation thresholds a few times higher; proton thresholds are higher still. A threshold energy of $E_{\mathrm{th}}=2 \mathrm{MeV} / \mathrm{nucl}$ corresponds to a velocity of $v_{\mathrm{th}}=20000 \mathrm{~km} / \mathrm{s}$; the amount of mass ejected above this energy is roughly $M(v>$ $\left.v_{\text {th }}\right) \sim 0.001 M_{\odot}$, i.e., a tenth of a percent of the total ejecta. This material has a kinetic energy of order $4 \times 10^{48} \mathrm{erg}$, i.e., about $0.4 \%$ of the super- 
nova energy. This is to be compared with a typical efficiency of $\sim 1 \%$ for acceleration of galactic cosmic ray supernova shocks (e.g., Gaisser 1990). Since prompt ejecta have comparable energetics, it is plausible that their effects could be important.

We are thus interested in calculating the initial spectrum of particles and determining their nuclear interactions as they propagate away from the explosion. The initial spectrum is calculated as follows. Imagine as well that the star emits at total number $N_{i}$ of isotopes $i$. The spectrum is obtained from the calculations of the mass, velocity, and composition of supernova shells. Specifically, we know the velocity $v(M)$ and mass fraction $X_{i}(M)$ as a function of mass coordinate $M$. We want the number spectrum $\partial N_{i} / \partial E$ for each species $i$ of interest. We have, for the total number of particles,

$$
N_{i}=\int_{M_{\ell}}^{M_{\mathrm{h}}} \frac{d M_{i}}{A_{i} m_{\mathrm{p}}}=\int_{M_{\ell}}^{M_{\mathrm{h}}} d M \frac{X_{i}}{A_{i} m_{\mathrm{p}}}
$$

integrated over the ejected shells mass between the inner $M_{\ell}$ and outer $M_{\mathrm{h}}$ mass coordinates of the ejecta. But by definition we also have

$$
\begin{aligned}
N_{i} & \equiv \int_{E_{\ell}}^{E_{\mathrm{h}}} d E \frac{\partial N_{i}}{\partial E} \\
& =\int_{v_{\ell}}^{v_{\mathrm{h}}} d v \frac{d E}{d v} \frac{\partial N_{i}}{\partial E} \\
& =\int_{M_{\ell}}^{M_{\mathrm{h}}} d M \frac{d v}{d M} \frac{d E}{d v} \frac{\partial N_{i}}{\partial E}
\end{aligned}
$$

and so by equations (11) and (2) we have

$$
\frac{\partial N_{i}}{\partial E}=\frac{X_{i}}{A_{i}}\left(m_{\mathrm{p}} \frac{d v}{d M} \frac{d E}{d v}\right)^{-1}
$$

and in the nonrelativistic case which holds here, we may simplify further to

$$
\frac{\partial N_{i}}{\partial E}=\frac{1}{m_{\mathrm{p}}^{2}} \frac{X_{i}}{A_{i}} \frac{1}{v} \frac{d M}{d v}
$$

Now we may define and then estimate the rate of $\mathrm{LiBeB}$ or $\gamma$-ray production by this flux. Formally, the rate is given by

$$
\begin{aligned}
\frac{d N_{l}}{d t} & =\sum_{i j} n_{j} \int_{E_{\mathrm{i}}}^{\infty} d E \sigma_{i j}^{l} \frac{\partial N_{i}}{\partial E} v \\
& =\sum_{i j} n_{j} \frac{1}{A_{i} m_{\mathrm{p}}^{2}} \int_{E_{\mathrm{i}}}^{\infty} d E \sigma_{i j}^{l} X_{i} \frac{d M}{d v}
\end{aligned}
$$

Note that the spectrum $\partial N_{i} / \partial E$ will be out of equilibrium and so time dependent (in contrast to the steady state of galactic cosmic rays). Because of the energy losses, the fast particle flux will be continuously degraded in intensity and energy over time. For the purposes of a first approximation, however, imagine a constant flux lasting for a duration $\Delta t$. The appropriateness of this approximation will be discussed in $\$ 5$.

From eq. (5) it is clear that what is crucial to our calculation is the nature of the fastest ejecta, i.e., the composition $X_{i}$ and the velocity spectrum $d M / d v$ of the outermost supernova shells. Both of these will be sensitive to the progenitor mass and history. Specifically, the outermost composition (and indeed the pre-explosion mass) will depend on the amount of mass loss the progenitor has suffered. Although the outermost layers will be born with the composition of the ISM at the time, with sufficient mass loss this could be shed and the inner nucleosynthetic shells can be exposed. Thus mass loss considerations are crucial for determining the fast particle composition.

Since mass loss can change the progenitor mass considerably, it also affects the velocity of the ejecta, and thus influences the normalization of the velocity spectrum. However, it appears that the shape of the velocity spectrum is generically a power law to a reasonable approximation. In a beautiful analytic treatment, Nadyozhin (1994) has examined the passage of the type II supernova shock through the outermost layers. He shows that in this a self-similar solution holds, and calculates the velocity spectrum of the fastest layers. He finds the spectrum to 
have the form of a power law with a cutoff at a maximum velocity $v_{\max }$. Specifically, he finds

$M(v)=\left\{\begin{array}{cl}\mathcal{B}\left(v^{-s}-v_{\max }{ }^{-s}\right)+\Delta M, & v<v_{\max } \\ 0, & v \geq v_{\max }\end{array}\right.$

where $\mathcal{B}$ is a constant that depends on the details of the hydrodynamics, while $v_{\max }$ scales roughly as $v_{\max } \propto \sqrt{U / M_{\mathrm{ej}}}$ with the supernova mechanical energy $U$ and the ejected mass $M_{\text {ej }}$. For SN 1987A, Nadyozhin calculates $v_{\max }=(26-$ 40) $\times 10^{8} \mathrm{~cm} / \mathrm{s}$, corresponding to an energy (per nucleon) cutoff of $E_{\max }=6-8 \mathrm{MeV} /$ nucl.

As we will see in detail below, the presence of the cutoff velocity $v_{\max }$ is crucial in determining the nuclear interactions of the fast particles. Physically, the cutoff arises from the quenching of the shock's acceleration of the outer layers of the supernova. As the shock moves through the decreasing density of the outer atmosphere, the material is accelerated progressively faster in reaction to the large radiation pressure of the shock. This process ceases, however, when the shock approaches the surface and the optical depth for the shock radiation becomes comparable to the optical depth to reach the photosphere. At this point the radiation escapes from the putative supernova as an ultraviolet flash, and the shock causes no further direct acceleration (for further discussion see Nadyozhin 1994).

A very nice feature of the Nadyozhin spectrum is that it takes the exceedingly simple form of a power law. Thus one can immediately deduce that the effective particle spectrum (cf. eq. 5) is also a power law $d M / d v \propto v^{-(s+1)}$, or

$$
\frac{d M}{d v}(E) \propto E^{-(s+1) / 2}, \quad E<E_{\max }
$$

For SN 1987A, Nadyozhin estimates that $s=$ 7.2 , which gives an energy dependence of $E^{-4.1}$. This result is supported by Ensman \& Burrows

\footnotetext{
${ }^{1}$ Note there is an additional shell ejected at the highest velocity, but its mass is so small $\left(\Delta M \simeq 10^{-6} M_{\odot}\right)$ that we ignore it.
}

(1992) whose numerical model of SN 1987A was specifically designed to calculate shock breakout.

The slope $s$ of the velocity spectrum (eq. 6) is related to the hydrodynamic properties of the self-similar shock solution. In particular, $s=$ $(n+1) / \gamma$, where $n$ is a power law index for the density dependence on the distance to the surface, and $\lambda$ is a similar index for the velocity (related to $n$ and the adiabatic index). At any rate, the universality of the power law solution is very fortuitous, as it allows us to make general remarks about all SNe.

The other major feature of the velocity spectrum which is equally crucial for our purposes is the existence of the cutoff in velocity, and thus in energy. The cutoff is quite near to the $\gamma$-ray and spallation thresholds, and so will greatly emphasize the differences among the different thresholds. To be sure, there is the above-mentioned scaling with supernova mass and energy, so a lighter pre-supernova star could perhaps achieve higher energies. Regardlessly, the low energy behavior of the cross sections will be crucial. In particular, protons reactions are disallowed below $\sim 20 \mathrm{MeV} /$ nucl for $\mathrm{C}$ and $\mathrm{O}$ reactions, but since $\alpha$ thresholds are lower by a factor $\sim 4$ in energy per nucleon, the components of the ejecta in $\alpha$ particles, and heavier nuclei which can interact with interstellar He, will be crucial.

The smallness of the cutoff energy means that the numerical calculations are difficult to do correctly. Results are very sensitive to the generally unmeasured threshold behavior of the cross sections. Semianalytic fits to experimental data (Read \& Viola 1984) do extrapolate down to threshold, but clearly there is a need to understand this low-energy physics exceedingly well, and we urge experimentalists to consider these measurements. Indeed if accurate cross sections were available, one could turn the problem around, and use the $\gamma$-ray and spallation yields as a powerful diagnostic on the fast ejecta's spectrum and composition.

Another word of caution: Nadyozhin's calcu- 
lation was plainly not intended for the use we make of it. In particular, it is doubtful that the real cutoff behavior is as sharp as he calculates it to be (eq. 6). In actual supernovae it is likely that the quenching of the shock is a continuous process and so while the cutoff scale may be correct, the details of the spectrum at these highest energies may be quite complicated. One should thus regard numerical results obtained via eq. (6) as rough indicators of the yields one expects from these fast ejecta. Nevertheless, we forge ahead in applying our method to the recent $\gamma$-ray observations of Orion.

\section{Gamma-ray Observations of Orion}

The $\gamma$-ray production in the Orion complex is huge, as Bloemen et al. (1993) recently determined with the COMPTEL experiment on the Compton Gamma Ray Observatory. The most surprising feature of the $\gamma$ emission from Orion is that the large flux is confined to the $3-7 \mathrm{MeV}$ range. Bloemen et al. (1993) measured the flux in the $3-7 \mathrm{MeV}$ band to be

$\Phi_{\gamma}(3-7 \mathrm{MeV})=(1.0 \pm 0.15) \times 10^{-4} \mathrm{~cm}^{-2} \mathrm{~s}^{-1}$.

In this range, the spectrum shows features consistent with de-excitation emission from the 4.44 $\mathrm{MeV}$ excited state of ${ }^{12} \mathrm{C}^{*}$ and the $6.13 \mathrm{MeV}$ state of ${ }^{16} \mathrm{O}^{*}$; these features have roughly the same amplitude.

The features at 4 and $6 \mathrm{MeV}$ appear wide, which would have implications for the nature of the accelerated particles due to the kinematics of the nuclear excitations. Fast $\mathrm{C}$ and $\mathrm{O}$ nuclei give rise to fast ${ }^{12} \mathrm{C}^{*}$ and ${ }^{16} \mathrm{O}^{*}$ which have Dopplerbroadened decay lines, whereas fast protons and $\alpha$ nuclei lead to slow ${ }^{12} \mathrm{C}^{*}$ and ${ }^{16} \mathrm{O}^{*}$ having narrow lines. However, Bloemen et al. (1993) caution that their lineshapes are not corrected for broadening due to finite detector response, and to the widths are artificial, a point also noted by Reeves \& Prantzos (1995) and emphasized via detailed calculations by Ramaty, Kozlovsky, \&
Lingenfelter (1995a, 1995b).

The COMPTEL experiment also set limits to the flux outside of the $3-7 \mathrm{MeV}$ band; in particular, the flux in the $1-3 \mathrm{MeV}$ range was constrained to be $\lesssim 1 / 7$ that of the $3-7 \mathrm{MeV}$ range. The lack of such lines is a crucial indicator of the lack of fast protons or $\alpha$ particles, which would excite ambient $\mathrm{Fe}, \mathrm{Si}$, and $\mathrm{Ne}$, leading to emission in this range.

It is assumed that these lines have their origin in energetic particle interactions with ambient matter in the Orion complex, but it is clear that any explanation of the $\gamma$ emission will have to be unconventional. No lines are observed below the $3-7 \mathrm{MeV}$ band, and no excess $\pi^{0}$ decays have been observed at higher energies (i.e., no excess over the level expected from galactic cosmic rays; see Digel, Hunter, \& Mukherjee 1995). The if galactic cosmic rays produced the $3-7 \mathrm{MeV}$ lines, they would also produce $1-3 \mathrm{MeV} \gamma$-ray lines which are not observed; also the GCR intensity would have to be much larger, which would give a high excess of $\pi^{0}$ decay photons, again unobserved. Moreover, the energetic demand would be enormous. This process is therefore excluded.

The most economic way to obtain the observed $\gamma$-ray flux is through accelerated $\mathrm{C}$ and O nuclei, and above all, a suppression of protons (Ramaty, Kozlovsky, \& Lingenfelter 1995a, 1995b; Vangioni-Flam et al. 1996; Cassé, Lehoucq, \& Vangioni-Flam 1995; and Cassé et al. 1994). Indeed, if one assumes merely that the Orion $\gamma$-rays arise from energetic particle interactions, then one may deduce in a model-independent way the particles must be a large flux with low energy, predominantly composed of $\mathrm{C}$ and $\mathrm{O}$ rather than protons and $\alpha$ particles.

A low energy CO flux was suggested by the Bloemen et al. (1993) paper itself, and the low energy character was assumed in all subsequent efforts at explaining the $\gamma$-ray emission. Bykov \& Bloemen (1994) and Nath \& Biermann (1994) connect the observation to theories of cosmic ray origin. Bykov \& Bloemen (1994) propose a spe- 
cific spectrum arising from collisions between supernova shocks and stellar winds. They use pure supernova compositions in their calculations, but it remains unclear how much of an admixture of normal material will be present in the hot regions they consider. Nath \& Biermann (1994) favor acceleration in shocks of winds of hot $\mathrm{O}$ and B stars; they argue that this naturally gives the desired composition. Ramaty, Kozlovsky, \& Lingenfelter (1995a) pointed out that while the observed $\gamma$-ray features appear broad, there is insufficient experimental resolution to exclude a narrow line component. Parameterizing a low energy spectrum, they tried different source compositions, showing that a composition like that of a WC supernova wind would be needed to meet the $\gamma$ spectrum and energetic constraints. These authors have also suggested (Ramaty, Kozlovsky, \& Lingenfelter 1995b) that the low energy cosmic rays could have an origin akin to that of the socalled anomalous component of the cosmic rays observed at earth (a suggestion also made by Jin \& Clayton 1995).

A low energy cosmic ray flux has implications for the nucleosynthesis of $\mathrm{Li}, \mathrm{Be}$, and $\mathrm{B}$ (LiBeB), as first pointed out by Meneguzzi \& Reeves (1975b). Cassé, Lehoucq, \& VangioniFlam (1995) investigated the implications of such a process for $\mathrm{LiBeB}$ chemical evolution. They show that the LiBeB synthesis can be significant (for an exposure time of $10^{5} \mathrm{yr}$ ), comparable to that of galactic cosmic rays (e.g. Walker, Mathews, \& Viola 1985).

Much of the work on the Orion $\gamma$-rays thus far has either: (1) assumed arbitrary flux shape, or (2) assumed SN composition. These first approaches adopt a spectrum of the form $\propto E^{-s}$, with $s=0$ below an energy $E_{\mathrm{c}}$, and $n=5-10$ above $E_{\mathrm{c}}$ (a form chosen for exploratory purposes, following Meneguzzi \& Reeves (1975b) and Ramaty, Kozlovsky, \& Lingenfelter $(\overline{1979)})$ and try different supernova compositions, as Bykov \& Bloemen (1994). We propose a more specific scenario related to a type Ic supernova explosion in a molecular cloud.

Our mechanism naturally has all of the needed features, provided only that the supernova exploding within Orion be sufficiently massive to have undergone significant mass loss. Then the progenitor would be stripped to a CO core, which would be ejected at high speeds (for a fixed explosion energy, a smaller core mass has less gravitational binding energy and so leads to higher kinetic energy for the ejecta). Furthermore, neither the fast particle spectrum nor the number of particles is arbitrary, but rather these are given by the supernova model. Thus the predictions of this process are specific and so the mechanism is testable.

\section{Confrontation with the Orion Obser- vation}

We have seen that theory allows us to compute $\dot{N}_{\gamma}$, while the Orion observation report the flux $\Phi_{\gamma}$ at Earth. These quantities are simply related by

$$
\begin{aligned}
\frac{d N_{\gamma}}{d t} & =4 \pi R^{2} \Phi_{\gamma} \\
& =(3.0 \pm 0.45) \times 10^{39} \mathrm{~s}^{-1}
\end{aligned}
$$

using $R_{\text {Orion }} \simeq 500 \mathrm{pc}$ as the distance to Orion, and assuming the flux to be emitted isotropically so that the total subtended solid angle is $4 \pi$. Of course it is conceivable that the emission is anisotropic due to, e.g., magnetic fields having somehow focussed (or defocussed) the beam in our direction. In the lack of clear evidence for such effects, we will henceforth make the simplest assumption regarding the angular distribution of the radiation and consider it to be isotropic.

To get a feel for the numerical results, let us make an order of magnitude estimate of the $\gamma$ ray emissivity. The particles of interest are the ejecta having a velocity larger than the reaction thresholds; these particles have mass $M_{\mathrm{ej}}(v>$ $\left.v_{\text {th }}\right)$. Taking an energy $\left(E_{\min }=2 \mathrm{MeV} / \mathrm{nucl}\right)$ slightly above the threshold for gamma rays $(\sim$ 
1.5 $\mathrm{MeV}$ for $\alpha+\mathrm{C}$ ), we have

$$
\begin{aligned}
\dot{N}_{\gamma} \sim & n_{\text {Orion }} v \sigma_{\mathrm{pC}}^{\gamma} N_{\mathrm{CO}}\left(v>v_{\min }\right) \\
= & n_{\text {Orion }} v \sigma_{\mathrm{pC}}^{\gamma} \frac{X_{i}^{C O}}{A_{i}} \frac{M_{\mathrm{ej}}\left(v>v_{\text {min }}\right)}{m_{\mathrm{p}}} \\
= & 1.0 \times 10^{39} \mathrm{~s}^{-1} X_{\mathrm{C}} \\
& \times \frac{n_{\text {Orion }}}{20 \mathrm{~cm}^{-3}} \frac{\sigma_{\mathrm{pC}}^{\gamma}}{10 \mathrm{mb}} \frac{M_{\mathrm{ej}}\left(v>v_{\text {min }}\right)}{0.03 M_{\odot}}
\end{aligned}
$$

where we have taken the cross section to be a typical one in the low energy range, about $10 \%$ of the maximum, and have allowed for an average density in Orion that is moderately enhanced over that of the ISM.

Note that eq. (10) shows that for the fiducial values we have chosen, the emissivity is just at the observed levels for the $\mathrm{C}$ lines (which contribute about half of the total emissivity) but only if $X_{\mathrm{C}}$ (and similarly $X_{\mathrm{O}}$ ) is close to unity. That this, the fast ejecta must be predominantly composed of $\mathrm{C}$ and $\mathrm{O}$. But the fastest ejecta arises from material at the outermost layers of the supernova progenitor. One expects a "normal" supernova progenitor, one not having significant mass loss, to have a composition similar to that of the local ISM at the time of the progenitor's birth. This would give $\mathrm{C}$ and $\mathrm{O}$ mass fractions $<10^{-2}$, which leads, via eq. (10), to a significant underproduction of ${ }^{12} \mathrm{C}^{*}$ and ${ }^{16} \mathrm{O}^{*}$ lines.

However, if the progenitor has suffered significant mass loss, or has lost its outer envelope to a companion star, then it is possible that the inner nucleosynthetic shells could be left at the outer layers of the progenitor. In this case the fastest ejecta would have a very nonsolar composition. In particular, if the progenitor is reduced to a $\mathrm{CO}$ core, then it would have $X_{\mathrm{C}} \simeq X_{\mathrm{O}} \simeq 0.5$; this is just the composition required to explain the Orion observations. While these nuclei dominate, there remain some $\alpha$ particles and also a small amout of $\mathrm{Ne}$ (having the form ${ }^{22} \mathrm{Ne}$ rather than ${ }^{20} \mathrm{Ne}$ ).

We thus suggest that the Orion $\gamma$-rays are the result of an explosion of a $\mathrm{CO}$ core supernova, similar to that observed recently in the type Ic supernova 1994I (Schmidt, Challis, \& Kirchner 1994: Clocchiatti, Brotherton, Harkness, \& Wheeler 1994). Nomoto et al. (1994) argued that the observed characteristics of this event are best understood in the context of a CO model arising from envelope loss to a companion (as opposed to mass loss of a single star). While such events are not the most common fates of massive stars, they are not so unlikely: Nomoto et al. estimate a ratio of type Ic to type II explosions of $\sim 10 \%$.

We now apply our formalism in more detail to the particular case of a $\mathrm{CO}$ core explosion. We use the results from the supernova model of Nomoto et al. (1994) (specifically the $v(M)$ and $X_{i}(M)$ relations) to generate the spectrum shown in Figure 1. As expected, most of the particles indeed lie at low energies, and the composition varies over energies reflecting the variation of the composition with mass shell. Since the reaction thresholds are all $\geq 2 \mathrm{MeV} / \mathrm{nucl}$, the only part of the flux relevant to our problem is that at and above this scale. At these energies, the composition does not vary with radius (being that of the outmost shells). Moreover, the spectrum has, fortunately, settled down to a fairly smooth shape close to a power law, with a slope of approximately $\sim 4$, in reasonable agreement with the Nadyozhin analytical calculation. Preliminary calculations using the velocity profiles for Wolf-Rayet supernovae (Woosley, Langer, \& Weaver 1993) give a similar slope and so give further credence to the analytic solutions.

To investigate the effect of the flux, it is crucial to know its behavior for energies above those specified in the Nomoto calculation. We have chosen to extrapolate the flux to higher energies by taking a power law with the slope of the last points. Specifically, if we write $\phi \sim E^{-\alpha}$, we find $\alpha \sim 3.5$ around $1 \mathrm{MeV}$, steepening to $\alpha \sim 4$ around $2 \mathrm{MeV}$; it is this last slope we used in the extrapolation.

Following Nadyozhin, we wish to introduce a 
maximum energy $E_{\max }$ to the flux. The selfsimilar solution provides the scaling mentioned above, $E_{\max } \propto\left(U / M_{\mathrm{ej}}\right)^{1 / 2}$, which Nadyozhin normalizes to SN1987A hydrodynamic models. Scaling his result to our CO core explosion, and assuming a total energy $U=10^{51} \mathrm{erg}$, gives $E_{\max } \sim 40 \mathrm{MeV} /$ nucl. However, since the details of determining $E_{\max }$ are difficult, the supernova energy is uncertain as well, we will take $E_{\max }$ to be a parameter and we will investigate values in the range $8 \mathrm{MeV} /$ nucl $\leq E_{\max } \leq 50 \mathrm{MeV} /$ nucl.

Using this extrapolated flux we have evaluated numerically the ${ }^{12} \mathrm{C}^{*},{ }^{16} \mathrm{O}^{*},{ }^{22} \mathrm{Ne}^{*}$, and $\mathrm{LiBeB}$ production. We assume that the escape length $\Lambda_{\text {esc }}$ is large and so the LiBeB produced are all either thermalized through ionization losses (the dominant process), or lost through inelastic collisions. In fact, since the ionization losses dominate, the results are insensitive to the escape length. Further we assume that the medium has a solar system composition as given by Anders \& Grevesse (1989).

Given these parameters, the ratios among the $\gamma$ and spallation rates are fixed; however, to compute absolute rates requires specification of the Orion $\mathrm{H}$ density $n_{\mathrm{H}}$. Specifically, we find, for the total $\gamma$ production from ${ }^{12} \mathrm{C}^{*}+{ }^{16} \mathrm{O}^{*}$, we have

$$
\frac{d N_{\gamma}}{d t}=2.4 \times 10^{38} \frac{n_{\mathrm{H}}}{1 \mathrm{~cm}^{-3}} \text { photons } \mathrm{s}^{-1}
$$

with very little variation over the chosen range of $E_{\max }$. Comparing this to the measured value (eq. 9), we see that our mechanism can account for the observed Orion $\gamma$-ray flux if the ejecta encounter an average density

$$
n_{\mathrm{H}} \simeq 12 \mathrm{~cm}^{-3} \text {. }
$$

This is to be compared with to the estimated average density of Orion, $n_{\mathrm{H}}^{\text {Orion }} \simeq 5 \mathrm{~cm}^{-3}$ (Goudis 1982), the uncertainty in which is large enough so that it is in good agreement with eq. (12). We note, however, that the density requirement rises with the time since the explosion, since the flux is constantly decreasing in intensity. As discussed below (\$5.) the timescale for significant loss is energy-dependent but at least of order $1 \mathrm{kyr}$.

Figure 2(a) shows calculated the emissivity from excited ${ }^{12} \mathrm{C},{ }^{16} \mathrm{O}$, as well as ${ }^{22} \mathrm{Ne}$, plotted as a function of $E_{\max }$. The normalization is fixed by requiring the total ${ }^{12} \mathrm{C}^{*}+{ }^{16} \mathrm{O}^{*}$ emissivity to match the Orion observations (eq. 9); as discussed in the previous paragraph, this fixes a value of $n_{\mathrm{H}}$ for each $E_{\max }$, which appears in Figure 2(b). We see that over the preferred range of $E_{\max }$, the $\gamma$-line ratios, and the required density, show almost no variation, and what little change there is has settled down by $10 \mathrm{MeV} /$ nucl. We find $\mathrm{C}^{*} / \mathrm{O}^{*}=2.7$, consistent with the Bloemen et al. 1993 observations.

It is insufficient to show that the flux can produce the observed $3-7 \mathrm{MeV}$ lines; we must also show there will not be lines outside this range. In particular, we must show that the $1-3 \mathrm{MeV}$ lines are below the COMPTEL limits (c.f. 33 .). Here again, the composition of the stripped supernova core proves advantageous. In particular, not only are the fast ejecta dominant in $\mathrm{C}$ and $\mathrm{O}$, as discussed above, but they also have relatively small abundances of heavier elements. In particular, the next most abundant element, after C, O, and $\mathrm{He}$, is ${ }^{22} \mathrm{Ne}$. Although its abundance is not large, it is much larger than its solar proportion, indeed much larger than the solar proportion of heavy elements. Thus this will be the most important contributor of heavy element lines, even more than those caused by the $\alpha$ reactions in the ISM.

Indeed, we find $\left(\mathrm{C}^{*}+\mathrm{O}^{*}\right) / \mathrm{Ne}^{*} \gtrsim 13$ for all values of $E_{\max }$, which falls safely below the observational limits, which are of order 7 . However, if ours is the mechanism for making the $\mathrm{C}$ and $\mathrm{O}$ $\gamma$-rays, then there should be ${ }^{22} \mathrm{Ne}$ lines present at a level just below the current limits. While COMPTEL may not have a good chance to see them, INTEGRAL will see them in detail. This will then provide a clean discrimination between our idea and others, as our prediction of ${ }^{22} \mathrm{Ne}$ lines (at $1.3 \mathrm{MeV}$ ), rather than ${ }^{20} \mathrm{Ne}$ lines (at 1.6 
$\mathrm{MeV}$ ), is likely to be unique.

Given the roughness of our estimate, and its closeness to the observational limit, it is clear that the $1-3 \mathrm{MeV}$ window offers an important constraint. More detailed modeling is needed and is under way (Ramaty, Vangioni-Flam, Fields, \& Cassé 1996). Indeed, we see that the generic sensitivity of the $\gamma$-ray emission to the supernova composition and velocity structure makes the $\gamma$ spectrum a powerful diagnostic tool. Since all supernovae should have fast ejecta, one could in general turn the problem around and use the constraint as a probe of supernova ejecta.

Although a full study of fast particle interactions from all possible supernovae is beyond the scope of this paper, nevertheless some discussion of model dependence is in order. We are interested in the sensitivity to progenitor mass and mass loss, as manifested in the flux intensity, spectrum, and composition. To begin to address this question we have studied variants to the supernova model we have used thus far.

The hydrodynamics of the explosion determines the intensity and spectrum, and the Nadyozhin (1994) model suggests (eq. 6) that the spectral slope is fairly independent of the initial mass, but the velocity scaling and energy cutoff depends on $v_{\max } \propto \sqrt{U / M_{\mathrm{ej}}}$. The scaling with mass means that significant mass loss can be important since, for a fixed explosion energy, it reduces the gravitational potential to be overcome and so increases the final kinetic energy. To test the accuracy of the expected scaling with the explosion energy (at a fixed mass), we compare the spectrum for a $2.1 M_{\odot}$ core at energies of $(0.6,0.8,1) \times 10^{51} \mathrm{erg}$ (Nomoto et al. 1995a, 1995b). Results appear in figure 3; we see that while the scaling is not exact, it is a good approximation. Note that changes in energy scaling do not affect the intensity of the scaled flux, but the shifts in the velocity scale change the intensity of the relevant, highest energy flux.

To test the accuracy of the expected scaling with progenitor mass (for a fixed explosion energy), we compare the spectrum from the $2.1 M_{\odot}$ $\mathrm{CO}$ core we have considered so far with that of $\mathrm{CO}$ cores with masses 1.8 (Iwamoto et al. 1994) and $2.9 M_{\odot}$ (Nomoto et al. 1995a). The results appear in figure 4. Here we find the agreement is not as good; indeed it appears that the particle fluxes are more similar without the scaling. [f While for our calculations we have used the numerical results anyway, it would be interesting if the results were less sensitive to ejecta mass than expected, as this would lead to larger fluxes (above threshold) than expected. At any rate we find that as a whole, the slope, the energy cutoff, and the intensity is well-described by the very simple Nadyozhin model itself coming from well-understood physics.

We now turn to the composition, which depends strongly on the mass loss, and so is tied to much less certain physics. Indeed, mass loss is poorly understood and simply treated parametrically. We have tried using different mass loss rates; results appear in Table 7. At any rate, it is apparent from figure 1, the composition of the fastest shells is fairly homogeneous. Thus one need only determine the (uncertain) outermost composition without having to get the full profile and convolving it with the hydrodynamics.

\section{Propagation and Energetics}

Recall that in calculating the rates for nuclear interactions of the fast particles we have assumed the flux to be present at full strength for some time interval $\Delta t$, during which the spectrum is constant. While this is adequate for estimates of emissivity, it is too simplistic. At the energies of interest the particles are stopped predominantly by ionization energy losses. As these losses are a strong function of energy and of $A$ and $Z$ (scaling

\footnotetext{
${ }^{2}$ Note, however, that the plot only demonstrates the scaling of the overall intensity; the models on which the calculations are based do not include the physics of the shock breakout and so cannot show the energy cutoff.
} 
as $\left.Z^{2} / A\right)$, the particle spectrum and composition will change with time.

The calculation is done in detail in Fields (1996). The basic effect is that the spectrum continually becomes less intense, but also harder. To see this, imagine an (unphysical) spectrum that is a $\delta$-function in energy. It would degrade from its initial energy according to the usual BetheBloch $\partial E / \partial t$ formula. Thus for a continuous spectrum, one expects the entire flux to degrade in energy. But since $\partial E / \partial t \propto E^{-1}$, lower energy particles lose energy faster, so the spectrum, though less intense. has an increasing average energy per particle.

An important related point is that the stopping ranges are larger than size of Orion. This means that if the fast particles are still in Orion then the supernova is young. More quantitatively, the range of a $2 \mathrm{MeV} /$ nucl oxygen nucleus in Orion is about $50 \mathrm{pc}$; it traverses this distance in about 2 kyr; this is about a factor 3 smaller than the crossing time, but it is also a lower bound; for particles above about $5 \mathrm{MeV} / \mathrm{nucl}$ the range is larger than Orion. Thus we can use the crossing time to put an approximate upper limit to the supernova date of $\sim 10^{4}$ yr ago. Of course, an accurate calculation must include the fact that the exposure time is not the total stopping time but rather the time it takes the fast particles to pass below the threshold for $\gamma$-ray production. Since the stopping time is a strong function of energy $\left(t \propto E^{2}\right)$ this difference will only be important for the particles very close to the threshold energy. This effect is discussed in Fields (1996).

The possibility of a relatively recent supernova is constrained by observations of short-lived radioactivities. In particular, the lack of observed ${ }^{44} \mathrm{Ti}(\tau=78.2 \mathrm{yr})$ sets a lower limit to the supernova age of order $\tau$, assuming that there is significant ${ }^{44} \mathrm{Ti}$ production in the supernova in question. In fact, we can make this point more quantitative. The $\mathrm{CO}$ core supernova models we have been using give ${ }^{44} \mathrm{Ti}$ yields of order
$M_{44}=5 \times 10^{-5} M_{\odot}-2 \times 10^{-4} M_{\odot}$ (Nomoto et al. 1991; Kumagai et al. 1991; Thielemann et al. 1996), a value close to the $10^{-4} M_{\odot}$ inferred from observations in Cas A (Iyudin et al. 1994). If the time since the explosion is $\Delta t$, there will be a ${ }^{44} \mathrm{Ti}$ emissivity of

$$
\dot{N}_{44}=\frac{1}{\tau} \frac{M_{44}}{44 m_{\mathrm{p}}} e^{-\Delta t / \tau}
$$

at an energy $E_{\gamma}=1.16 \mathrm{MeV}$. The observational limit on such lines in Orion is $L \lesssim 3 \times$ $10^{38}$ photons s$^{-1}$ (namely the limit for the $1-3$ $\mathrm{MeV}$ band), which gives

$$
\Delta t \gtrsim \tau \ln \frac{M_{44}}{44 m_{\mathrm{p}} \tau L}=570 \mathrm{yr} .
$$

This is a strong constraint, being only a factor of 4 shorter than the minimum particle stopping time. Taking this result at face value, we are led to infer a possible age range of $0.6-10 \mathrm{kyr}$ for the supernova.

However, caution is merited in using the ${ }^{44} \mathrm{Ti}$ lower bound on the age. The ${ }^{44} \mathrm{Ti}$ yield is very uncertain, as it requires an accurate knowledge of which of the deepest layers of the star are ejected, and which fall back into the neutron star (i.e., the mass cut). Different mass cut choices are allowed and can lead to smaller ${ }^{44} \mathrm{Ti}$ yields (although the age limit depends only logarithmically on the mass yield). Also, we note that the observational limit we have used is that of COMPTEL and is more suited for broad lines; Bloemen et al. (1993) caution that narrow lines may be left undetected. And in any case, if the age is closer to the $\sim 10^{4}$ yr crossing time, then there is no ${ }^{44} \mathrm{Ti}$ to be seen by either COMPTEL or INTEGRAL. On the other hand, to take more optimistic viewpoint and turn the problem around: if there has been a very recent supernova in Orion, the ${ }^{44} \mathrm{Ti}$ lines (or lack thereof) can give important information about the mass cut.

Finally, we turn to the question of energy requirements. As pointed out by Ramaty, Kozlovsky, \& Lingenfelter (1995a, 1995b), the significant low-energy flux in Orion is a challenge 
to explain in a way that is energetically economical. These authors note that a flux lasting long enough ( $\left.\gtrsim 10^{5} \mathrm{yr}\right)$ to produce significant amounts of $\mathrm{Li}, \mathrm{Be}$, and $\mathrm{B}$ must be composed of relatively energetic particles ( $\gtrsim 30 \mathrm{MeV} /$ nucl) to avoid an unreasonable energy demand.

Our mechanism also must be shown to have reasonable energy requirements. To see most simply that indeed the energetics are acceptable, recall that our particles have a very definite source: one $\mathrm{C}+\mathrm{O}$ core supernova. Thus in this model the total energy required to accelerate the particles and thus create the observed $\gamma$-rays is exactly the (mechanical) energy of the supernova: $10^{51} \mathrm{erg}$. We note again that the flux intensity and composition of the particles is fully determined by the supernova model, generally and in the specific scenario we have adopted for Orion. The energy is always fixed to be that of the supernova, and so as a consequence we are not free to adjust the number of fast particles; thus the energy budget is automatically that of one supernova, but the resulting flux may or may not be observable (depending notably on the ejected mass).

The number of $\gamma$-rays per unit energy injected in particles has been calculated by other groups and so provides a useful quantity for comparison of the results. In our case, the total energy injected in fast particles is above $2 \mathrm{MeV} / \mathrm{nucl}$ is $W=1.4 \times 10^{50} \mathrm{erg}$, about $14 \%$ of the total energy (this is larger than the generic estimate given in the beginning of $\$ 2$. due to the unusually low ejecta mass). The total number of $\gamma$-rays made in Orion is the product of the present total emissivity and the irradiation time: $N_{\gamma}=\dot{N}_{\gamma} \tau \simeq 10^{50}$ photons (using a conservative exposure time of $\tau=1 \mathrm{kyr}$ ). This gives an efficiency $N_{\gamma} / W \simeq 0.7$ photon/erg. By way of comparision, Ramaty, Kozlovsky, \& Lingenfelter (1995a, 1995b) find values around 10 photons/erg, using a spectrum with a characteristic energy of $30 \mathrm{MeV} / \mathrm{nucl}$, which is in fact more efficient.
The implications in this difference in efficiencies can be understood by noting that the fast particle propagation is at all not the same in the two models. We do not use a steady state flux given by a thick target solution, but rater an impulsive flux. As a result, in our case the exposure time is fixed as we have just discussed, and is in fact much smaller that that of Ramaty et al. (1995a, 1995b) or Cassé, Lehoucq, \& VangioniFlam (1995). As the flux has a shorter duration its total energy requirements are less; on the other hand, it also produces less LiBeB, as we now see.

\section{Li, Be, and B Production}

With the above discussion one can compute the time-integrated production rates. The formalism and discussion is found in Fields (1996). The most important point is that the nuclear interaction rate proportional to $n_{\mathrm{ISM}}$ (cf. eq. 5), but the exposure is set by the stopping time which is proportional to $n_{\mathrm{ISM}}{ }^{-1}$. Thus the production at a given value of energy is related only to the stopping power; specifically, it is proportional to the ionization range as expressed in $\mathrm{g}$ $\mathrm{cm}^{-2}$. Roughly speaking, one integrates over ionization range rather than over time, to arrive at a yield that is a given number or mass of $\mathrm{LiBeB}$ per supernova.

Thus the absolute yields of $\mathrm{LiBeB}$ are independent of the density of the circumstellar medium; they are only dependent on the composition of the ISM. Consequently, for a fixed composition, the yield for a given supernova is independent of the region in which it explodes. However, while the same argument holds for the total number of $\gamma$-rays, their intensity does depend on $n$, as we have discussed above (cf. eq. 12). Note also that the composition of the medium, and of the ejecta, is crucial.

We have calculated the $\mathrm{LiBeB}$ yields for the ISM with a solar system composition. In figure 5 we plot the total mass of $\mathrm{LiBeB}$ isotopes pro- 
duced as a function of $E_{\max }$. We see that, unlike the results for $\gamma$-ray emission, the LiBeB production is very sensitive to $E_{\max }$. This may be understood very simply in terms of cross section thresholds. Typically, for spallation processes one has $E_{\mathrm{th}}(\alpha+\mathrm{N})<E_{\mathrm{th}}(\alpha+\mathrm{C})<E_{\mathrm{th}}(\alpha+\mathrm{O})$, so the low $E_{\max }$ behavior arises from the $\alpha+\mathrm{N}$ reactions, and the rapid variations happen when $E_{\max }$ rises above the $\alpha$ thresholds.

The implications of this mechanism for chemical evolution of $\mathrm{LiBeB}$ are hard to foresee given the yields for just one supernova type (and a special one at that). Nevertheless, it is of interest to compare to other suggested LiBeB production mechanisms in supernovae, e.g. the $\nu$ process yields (Woosley \& Weaver 1995) ${ }^{3}$. These are a function of supernova mass and metallicity, but at solar metallicity ${ }^{11} \mathrm{~B}$ production lies in the range $M_{\mathrm{B}} \simeq(1-30) \times 10^{-5} M_{\odot}$. It has been shown (Olive et al. 1994; Timmes, Woosley, \& Weaver 1995) that these yields, when combined with the usual GCR production of $\mathrm{LiBeB}$, are sufficient to explain the solar ${ }^{11} \mathrm{~B} /{ }^{10} \mathrm{~B}$ ratio (but not $\left.{ }^{9} \mathrm{Be}\right)$. Thus it would seem that since the $\nu$ process yields are significantly higher than that for our particular supernova, it is unlikely that our mechanism can produce the solar boron. A more detailed analysis of the relative LiBeB contributions of these processes as well as GCR will appear in a forthcoming paper (Vangioni-Flam et al. 1996).

While the supernova precursors appear to have trouble explaining the solar B abundances, it remains however to be seen if they may explain the Pop II abundances. A useful diagnostic is the $\mathrm{B} / \mathrm{O}$ ratio. For the $\mathrm{CO}$ supernova we have considered, the $\mathrm{O}$ production is $M_{\mathrm{O}} \simeq 0.2-0.4 M_{\odot}$

${ }^{3}$ In fact, other mechanisms of supernova production of LiBeB have been suggested, most notably that of Colgate (1973) and Dearborn et al. 1989. Both of these involve synthesis within the shock itself, as opposed to our treatment of the fast particles' interaction with the ISM. Each has also been shown to have apparently fatal problems, by Weaver (1976) and Brown et al. (1991), respectively.
(Thielemann et al. 1994, 1996). This gives, for high $E_{\max }, \mathrm{B} / \mathrm{O} \simeq 3 \times 10^{-7}$ by number. Comparing this with the solar ratio $(\mathrm{B} / \mathrm{O}) \odot=1.2 \times 10^{-6}$, we see that even if all of the solar $\mathrm{O}$ were produced by $\mathrm{CO}$ supernovae, the most favorable assumption, we still find B production to fall short by a factor of $2-3$, again with realistic $\mathrm{LiBeB}$ production giving smaller numbers. However, in Population II, we have $\mathrm{B} / \mathrm{O} \sim 0.1(\mathrm{~B} / \mathrm{O})_{\odot} \sim$ $(3-6) \times 10^{-7}$. Thus we would require only $\sim 5-10 \%$ of Pop II supernovae to be of this type in order to have significant Pop II B production by our mechanism; this is to be compared to the supernova type Ic/II ratio of $\sim 10 \%$ (Nomoto et al. 1994).

This suggests that the Pop II B (as well as Li and $\mathrm{Be}$ ) may have arisen from supernova precursors. If so, then since the fast particles are $\mathrm{C}$ and $\mathrm{O}$, then there is a natural explanation of the observed correlation of $\mathrm{Be}, \mathrm{B} \propto \mathrm{O}$ in Pop II stars, i.e. a linear slope (rather than the quadratic slope naively expected from galactic cosmic ray production). This possibility is intriguing and demands further investigation (Vangioni-Flam et al. 1996).

Regardless of the impact of our LiBeB yields on galactic chemical evolution, the production is still high enough to create large local enhancements of these elements. This is particularly important if the fast particles are well-confined by local magnetic fields and so the yields are concentrated in a small volume. Indeed, if sufficiently localized, the fast particle yields would dominate the local LiBeB abundances and ratios. This possibility is interesting in light of the Lemoine, Ferlet, \& Vidal-Madjar (1993) observation of a very localized Li isotopic anomaly in the direction of $\zeta$ Oph. The observed ${ }^{7} \mathrm{Li} /{ }^{6} \mathrm{Li}$ ratio is $\sim 2$ in one of the two observed components; this is tantalizingly close to the high $E_{\text {max }}$ values for this ratio found in figure 6. If this is so, then the other elemental and isotopic ratios in this region should be as given in the figure; in fact, none of these are very different from the solar ratios. Thus the sig- 
nature of supernova precursor production would be for the other ratios to appear solar, and of course, for the ${ }^{7} \mathrm{Li} /{ }^{6} \mathrm{Li}$ measurement to hold up.

Finally, if the process we discuss does produce significant Pop II ${ }^{6} \mathrm{Li}, \mathrm{Be}$, and $\mathrm{B}$, then one consequence is apparently that there are at least two other important sources of these. Galactic cosmic rays are an inevitable and significant source; furthermore, their inability to reproduce the ${ }^{11} \mathrm{~B} /{ }^{10} \mathrm{~B}$ ratio suggests the need for another mechanism as well. Perhaps the $\nu$-process is the remaining source, perhaps some other low-energy particle spectrum (Cassé, Lehoucq, \& VangioniFlam 1995; Ramaty, Kozlovsky, \& Lingenfelter 1995a), or perhaps something else entirely. At any rate, in explaining $\mathrm{LiBeB}$ production and evolution, one seems to be faced at the moment with an embarrassment of riches-more theories exist than problems to solve. To resolve the issue will require not just more observational data but also more accurate theory.

\section{Discussion}

We find that the prompt ejecta from a supernova may be behind the curious $\gamma$-ray observations of Orion. This mechanism has the advantage of providing a definitive physical mechanism for a low-energy particle spectrum with a non-solar composition, as seems required by the observations. To explain the observation we require the progenitor of the responsible supernova to have been not a "normal," type II supernova with a small wind, but instead an exploding CO core star. This leads not only to the observed superabundance of low energy $\mathrm{CO}$ nuclei, but also predicts that there will be ${ }^{22} \mathrm{Ne}$ (rather than ${ }^{20} \mathrm{Ne}$ ) lines just below the current upper limits. Consequently, our hypothesis is testable.

Furthermore, via the analytical supernova shock models of Nadyozhin (1994), we see that this mechanism is generic to supernova explosions. There are always precursor particles preceding the bulk of the shock, and these particles in- evitably have nuclear interactions with their environment if $E_{\max }$ is sufficiently large. The $\gamma$ production could be a new probe of the supernova explosion, providing a detailed signature of the outermost shells of material. This could in principle be used as an independent means of determining supernova masses (Fields, Cassé, Nomoto, Schramm, \& Vangioni-Flam 1996).

The implications for LiBeB evolution are unclear at the moment and bear further investigation. While it is not clear that the $\mathrm{LiBeB}$ yields will play a major role in galactic chemical evolution, they nevertheless could account for large local variations in these elements. Thus this process could offer a potential explanation for the low ${ }^{7} \mathrm{Li} /{ }^{6} \mathrm{Li}$ ratio observed by Lemoine et al. (1993) in the direction of $\zeta$ Oph.

We caution that calculations we present here are rough. Some improvements can be implemented without too much difficulty. For example, we have treated the propagation of the flux in a sketchy manner, but as we indicate the full time dependence should be considered. Also, the flux first encounters the supernova progenitor's wind, and the effect of traversing the wind should be included. Both of these effects will be addressed in a future paper (Fields 1996).

Another uncertainty in the calculations arises from the lack of accurate cross section measurements for $\gamma$-ray and spallation processes occurring near threshold. We urge experimentalists to examine this behavior. Finally, there are some uncertainties to our calculations which are more difficult to address accurately. The most notable of these is the effect of a magnetic field on the propagation and stopping of the particles. A sizable field could for example lead to anisotropic emission of particles and so of $\gamma$-rays. Spiraling in magnetic fields will also shorten the total displacement of the fast particles from the supernova explosion site. These effects are however difficult to estimate.

Despite these problems, the precursor particles clearly have the potential to provide a new 
probe of supernova explosions. More work on this mechanism is in order.

We thank Dave Schramm, Jim Truran, Don Ellison, and Simon Swordy for stimulating discussions, and Robert Mochkovitch for sagacious advice and a careful reading of the manuscript. We especially thank Reuven Ramaty for pointing out the ${ }^{44} \mathrm{Ti}$ constraint. This material is based upon work supported by the North Atlantic Treaty Organization under a Grant awarded in 1994. EV-F and MC are supported in part by PICS $n^{\circ} 114$ of the CNRS (Origin and Evolution of the Light Elements).

Since this work was originally submitted, we became aware of the Letter of Cameron et al. (1995), which suggests a type Ib supernova as a source of the fast particles in Orion. 
Table 1: Composition of outermost shells, by number

\begin{tabular}{ccccccc}
\hline \hline Iniitial Mass & CO core mass & Ejected mass & $\mathrm{He}$ & ${ }^{12} \mathrm{C}$ & ${ }^{16} \mathrm{O}$ & ${ }^{22} \mathrm{Ne}$ \\
\hline $13 M_{\odot}$ & $1.8 M_{\odot}$ & $0.54 M_{\odot}$ & 1.44 & 1.39 & $\equiv 1$ & 0.0203 \\
$15 M_{\odot}$ & $2.1 M_{\odot}$ & $0.86 M_{\odot}$ & 1.61 & 1.38 & $\equiv 1$ & 0.0337 \\
$18 M_{\odot}$ & $2.9 M_{\odot}$ & $1.54 M_{\odot}$ & 684 & 6.42 & $\equiv 1$ & 1.26 \\
\hline
\end{tabular}




\section{REFERENCES}

Anders, E., \& Grevesse, N. 1989, Geochim. et Cosmochim. Acta, 53, 197

Blanford, R., \& Eichler, D., 1987, Phys. Rep., 154,1

Bloemen, H., et al. 1993, A\&A, 281, L5

Brown, L.E., Dearborn, D.S., Schramm, D.N., Larsen, J.T., \& Kurokawa, S. 1991, ApJ, 371, 648

Bykov, A., \& Bloemen, H. 1994, A\&A, 283, L1

Cameron, A.G.W., Höflich, P., Meyers, P.C. \& Clayton, D.D. 1995, ApJ, 447, L53

Cassé, M., Fields, B.D., Nomoto, K., Schramm, D.N., \& Vangioni-Flam, E. 1996 in preparation

Cassé, M., \& Goret, H. 1978, ApJ, 221, 703

Cassé, M., Lehoucq, R., \& Vangioni-Flam, E. 1995, Nature, 373, 318

Cassé, M., Vangioni-Flam, E., Lehoucq, R., \& Oberto, Y. 1994, in Light Element Abundances, eds. P. Crane \& J. Faulkner, 389

Clocchiatti, A., Brotherton, M., Harkness, R.P., \& Wheeler, J.C. 1994, IAU Circ. No. 5972

Colgate, S.A. 1973, ApJ, 181, L53

Colgate, S.A., \& Johnson, M.H. 1960, Phys. Rev. Lett., 5, 235

Dearborn, D., Schramm, D.N., Steigman, G., \& Truran, J. 1989, ApJ, 347, 455

Digel, S.W., Hunter, S.D., Mukherjee, R. 1995, ApJ, 441, 270

Ensman, L., \& Burrows, A. 1992, ApJ, 393, 742

Jones, F.C., \& Ellison, D.C. 1991, Sp. Sci. Rev., 58,259
Fields, B.D. 1996 in preparation

Gaisser, T.K. 1995, Cosmic Rays and Particle Physics, (Cambridge: Cambridge University Press), 149

Goudis, C. 1982, The Orion Complex: A Case Study of Interstellar Matter (D. Ridel: Dodrecht), 12

Iwamoto, K., et al. 1994, ApJ, 437, 115

Iyudin et al. 1994, A\&A, 284, L1

Jin, L., \& Clayton, D.D. 1995, ApJ, 447, L53

Kumagai, S., Shigeyama, T., Hashimoto, M., \& Nomoto, K. 1991, A\&A, 243, L113

Lemoine, M., Ferlet, R., Vidal-Madjar, A. 1995, $\mathrm{A} \& \mathrm{~A}$, in press

Meneguzzi, M, \& Reeves, H. 1975a, A\&A, 40, 91

Meneguzzi, M, \& Reeves, H. 1975b, A\&A, 40, 99

Meyer, J.-P. 1985a, ApJS, 57, 151

Meyer, J.-P. 1975b, ApJS, 57, 173

Montmerle, T. 1977, ApJ, 217, 872

Nadyozhin, D.K. 1994, in Les Houches, Session LIV 1990: Supernovae, ed. S. Bludman, R. Mochkovitch, \& J. Zinn-Justin (Elsevier: New York), 569

Nath, B., \& Biermann, P. 1994, MNRAS, 270, L33

Nomoto, K., Kumagai, S., \& Shigeyama, T. 1991, in Gamma-Ray Line Astrophysics, eds., P. Durouchoux \& N. Prantzos (AIP 232: New York), 236

Nomoto, K., et al. 1994, Nature, 471, 227

Nomoto, K., et al. 1995a, in IAU Symposium 165, Compact Stars in Binaries, ed. J. van Paradij \& E.P.J. van den Heuvel (Kluwer: Dodrecht), in press 
Nomoto, K., et al. 1995b, in 17th Texas Symposium on Relativistic Astrophyiscs (Ann. N.Y. Acac. Sci.: New York), in press

Olive, K.A., Prantzos, N., Scully, S., \& VangioniFlam, E. 1994, ApJ, 424, 666

Ramaty, R., Kozlovsky, B, \& Lingenfelter, R.E. 1979, ApJS, 40, 487

Ramaty, R., Kozlovsky, B, \& Lingenfelter, R.E. 1995a, ApJ, 438, L21

Ramaty, R., Kozlovsky, B, \& Lingenfelter, R.E. 1995b, ApJ, 438, L21

Ramaty, R., Vangioni-Flam, E., Fields, B.D., \& Cassé, M. 1996, in preparation

Read, S.M., \& Viola, V.E. 1984, Atom. Data \& Nuc. Data Tables, 31, 359

Reeves, H., \& Prantzos, N. 1995, in The Light Element Abundances, eds. P. Crane \& J. Faulkner, 382

Schmidt, B., Challis, P., \& Kirchner, R. 1994, IAU Circ. No. 5966

Steigman, G., \& Walker, T.P. 1992, ApJ, 385, L13

Thielemann, F.-K., Nomoto, K., \& Hashimoto, M., 1994, in Supernovae, Les Houches, Session LIV, eds. S. Bludman, R. Mochkovitch, \& J. Zinn-Justin (Elsevier: Amsterdam), 629

Thielemann, F.-K., Nomoto, K., \& Hashimoto, M. 1996, ApJ, in press

Timmes, F.X., Woosley, S.E., \& Weaver, T.A. 1995, ApJ, in press

Vangioni-Flam, E., Olive, K.A., Fields, B.D., \& Cassé, M. 1996, ApJ sumitted

Walker, T.P., Mathews, G.J., \& Viola, V.E. 1985, ApJ, 299, 745

Weaver, T., 1976, ApJS, 32, 233
Woosley, S.E., Langer, N. \& Weaver, T.A. 1993, ApJ, 411, 823

Woosley, S.E., \& Weaver, T.A. 1995, ApJ, in preparation
This 2-column preprint was prepared with the AAS LATEX macros v3.0. 


\section{FIGURE CAPTIONS}

1. The spectrum of the ejecta from a $2.1 M_{\odot}$ $\mathrm{C}+\mathrm{O}$ core, for a progenitor originally of mass $15 M_{\odot}$. Note the absence of protons and the dominance of $\mathrm{C}$ and $\mathrm{O}$ over $\alpha$ particles. The small bounciness is due to numerical inaccuracy, but the large jumps, e.g. in the $\mathrm{C}$ spectrum, are real and arise from changes in the compositions of the various nuclear burning shells. In the energy range of interest $(E \gtrsim 1 \mathrm{MeV} / \mathrm{nucl})$, the spectrum is well approximated by a power law with a slope of $\sim 4$, in good agreement with the analytic results by Nadyozhin (1994). The endpoints of the curves at $E \sim 3.5 \mathrm{MeV}$ are artifacts of the supernova model and do not correspond to $E_{\max }$.

2. (a) ${ }^{12} \mathrm{C}^{*},{ }^{16} \mathrm{O}^{*}$, and ${ }^{22} \mathrm{Ne}^{*}$ emission as a function of $E_{\max }$. Curves are normalized so that the total $\mathrm{CO} \gamma$ emission matches the Bloemen et al. (1993) Orion observations, i.e., $\dot{N}\left(\mathrm{C}^{*}+\mathrm{O}^{*}\right)=3 \times 10^{39} \mathrm{~s}^{-1}$. This amounts to fixing the hydrogen density $n_{\mathrm{H}}$ at each $E_{\text {max }}$.

(b) The required hydrogen density $n_{\mathrm{H}}$ as a function of $E_{\max }$. Note that the density is fairly constant over $E_{\max }$, and is only modestly above the average Orion density. The rise at low $E_{\max }$ stems from the lower particle flux above $\gamma$-ray production energies.

3. (a) The spectrum (as in figure 11) for explosion energies $U=0.6 \times 10^{51}, 0.8 \times 10^{51}$, and $1 \times 10^{51} \mathrm{erg}$, all with CO core mass $2.1 M_{\odot}$. (b) As in (a), with the energies rescaled according to the rule $E^{*}=\left(U / 10^{51} \mathrm{erg}\right)^{-1} E$; for the analytic scaling law this should give identical spectra. While this is not perfectly true, clearly the scaling is a good approximation.
4. (a) The spectrum (as in figure 1) for $\mathrm{CO}$ core masses $1.8,2.1$, and $2.9 M_{\odot}$, all having an explosion energy of $U=1 \times 10^{51}$ erg. Although the shapes are similar, the higher energy scale for the lower masses means that they also have larger intensities in the energies above threshold.

(b) As in (a), with the energies rescaled according to the rule $E^{*}=\left(M / 2.1 M_{\odot}\right) E$; for the analytic scaling law this should give identical spectra and the curves should overlap. We see that this scaling is not very good, and the results seen more consistent with little or no mass dependence.

5. The yields of LiBeB isotopes as a function of the cutoff energy $E_{\max }$. The strong $E_{\text {max }}$ dependence is a result of crossing the various thresholds for spallation production. Note that the yields are very small, even though our CO core supernova must be considered as a best (and uncommon) case. Thus it seems unlikely that the LiBeB production by fast ejecta is significant for chemical evolution.

6. Elemental and isotopic ratios of $\mathrm{LiBeB}$ as a function of $E_{\max }$. The extreme behavior at low $E_{\max }$ is due to threshold behavior. Since the absolute yields are small, these ratios are only of interest if they may be observed in a very localized region. The strong variations at low $E_{\max }$ are due to cross section threshold and resonance effects. 


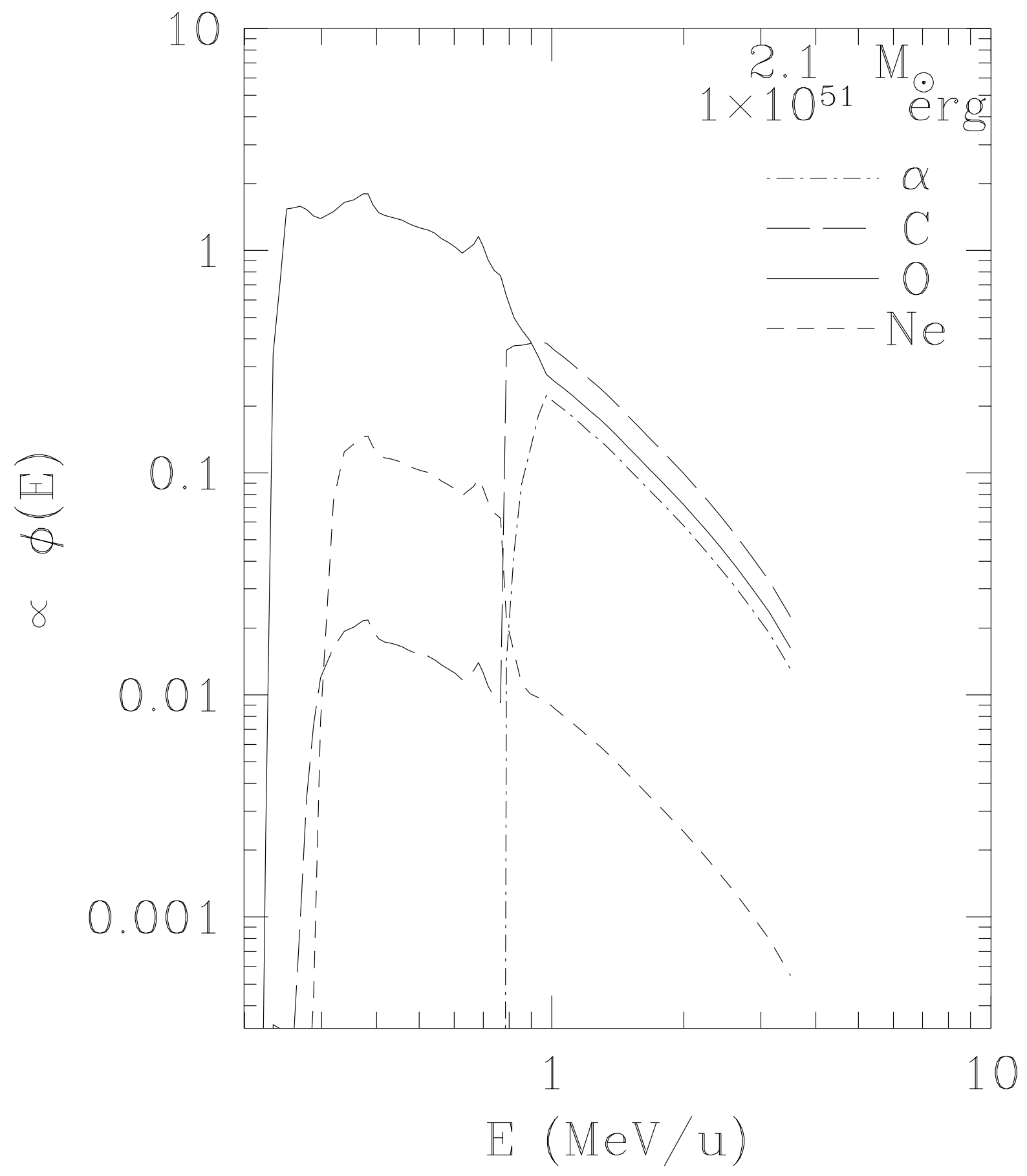



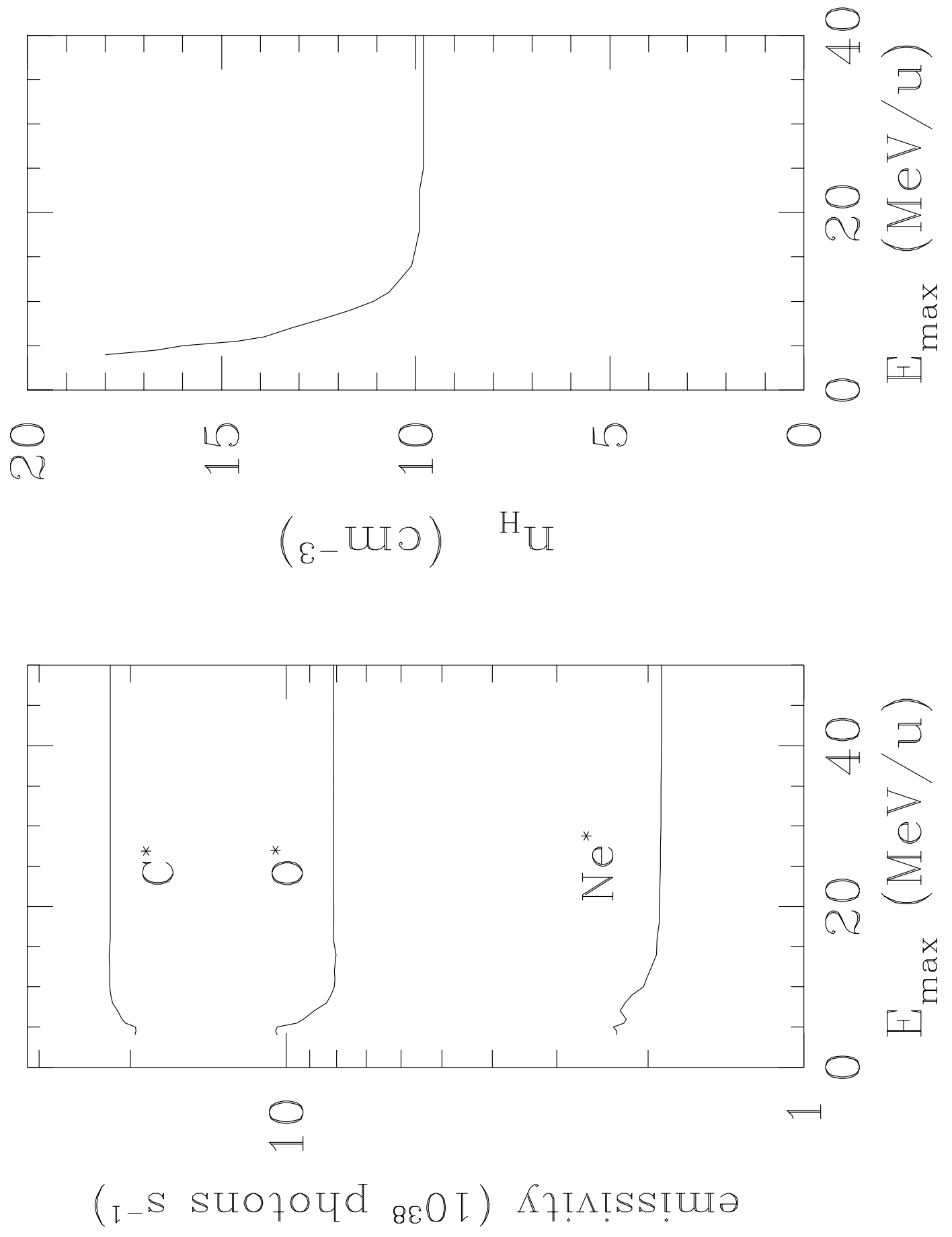

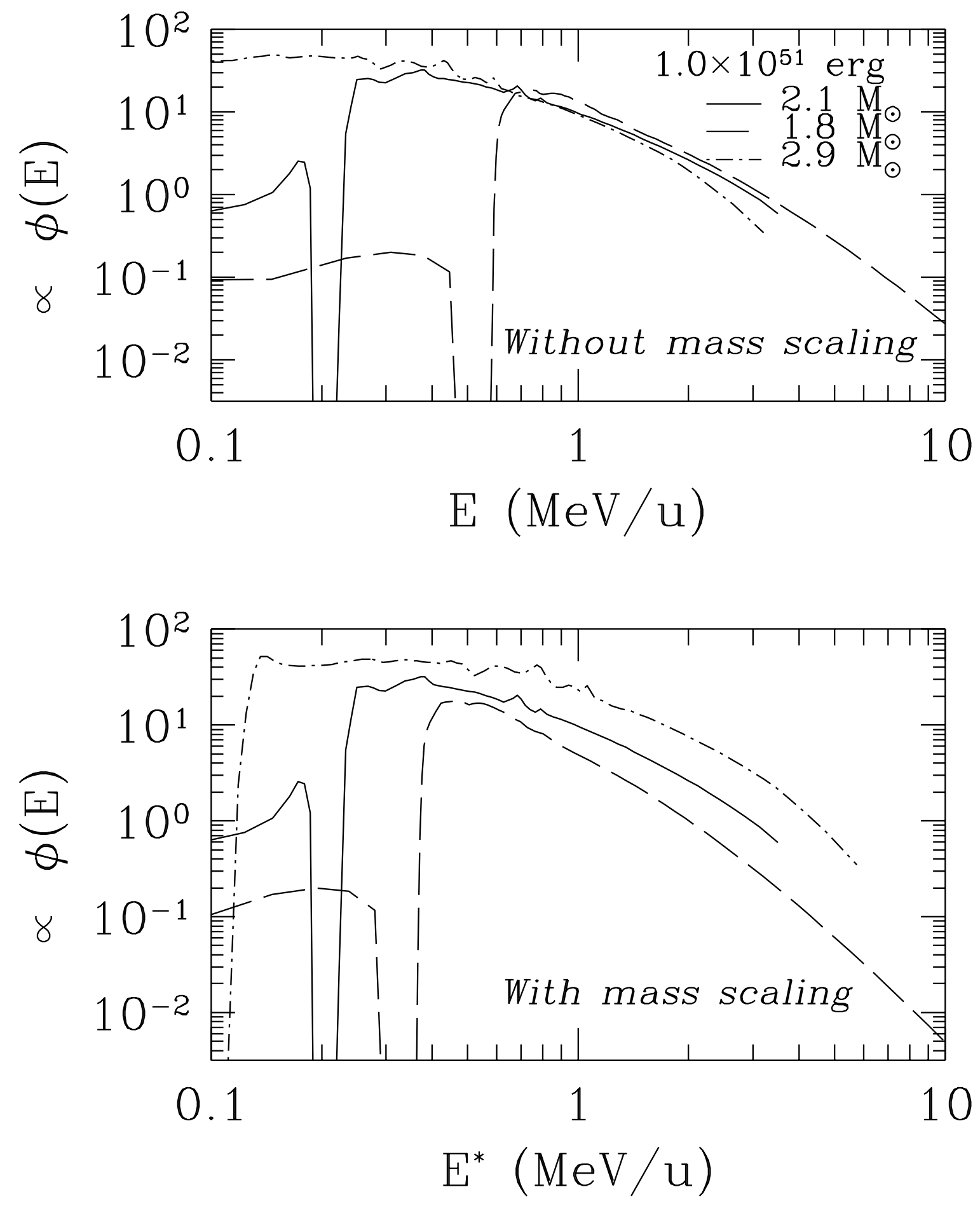

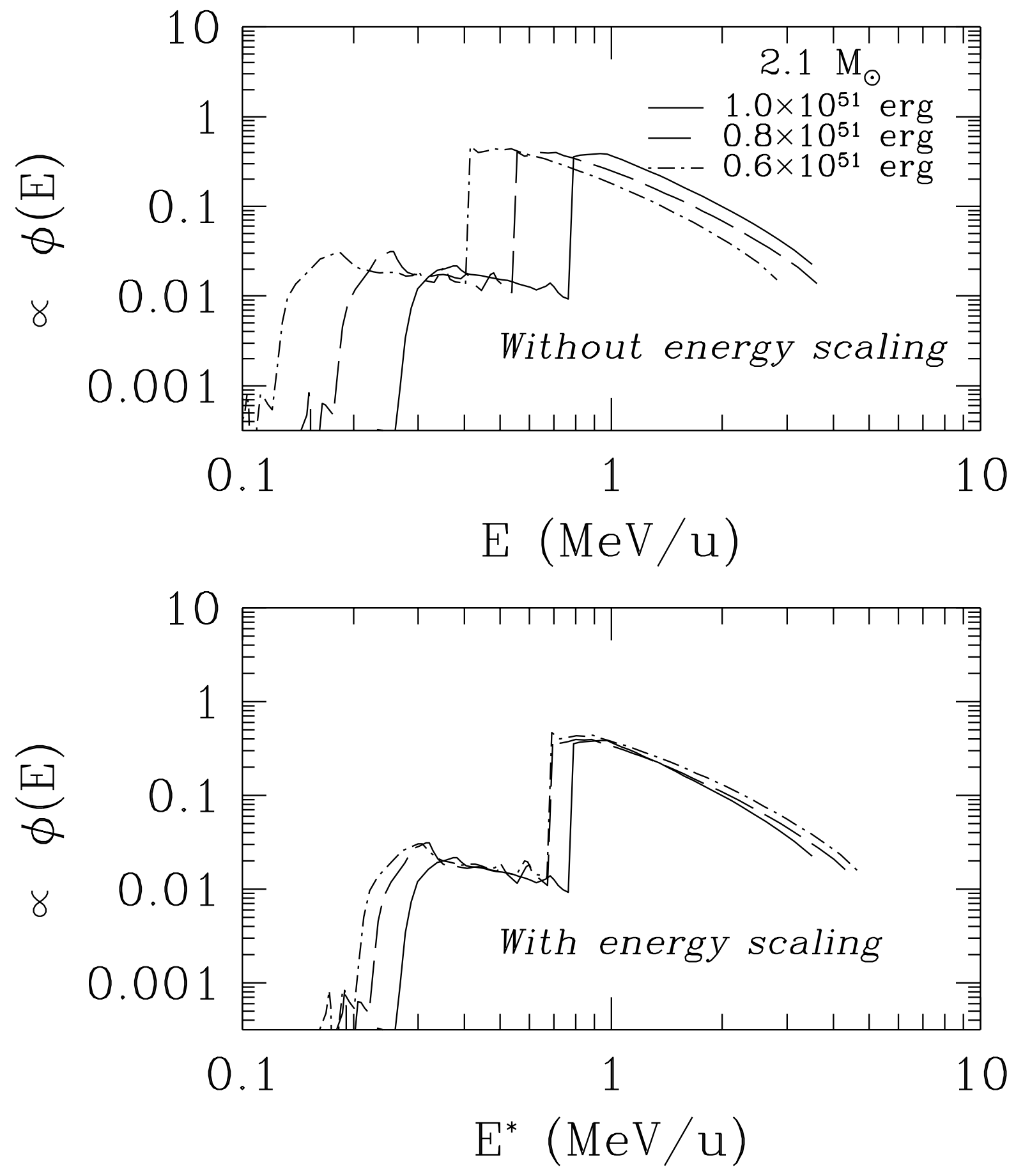

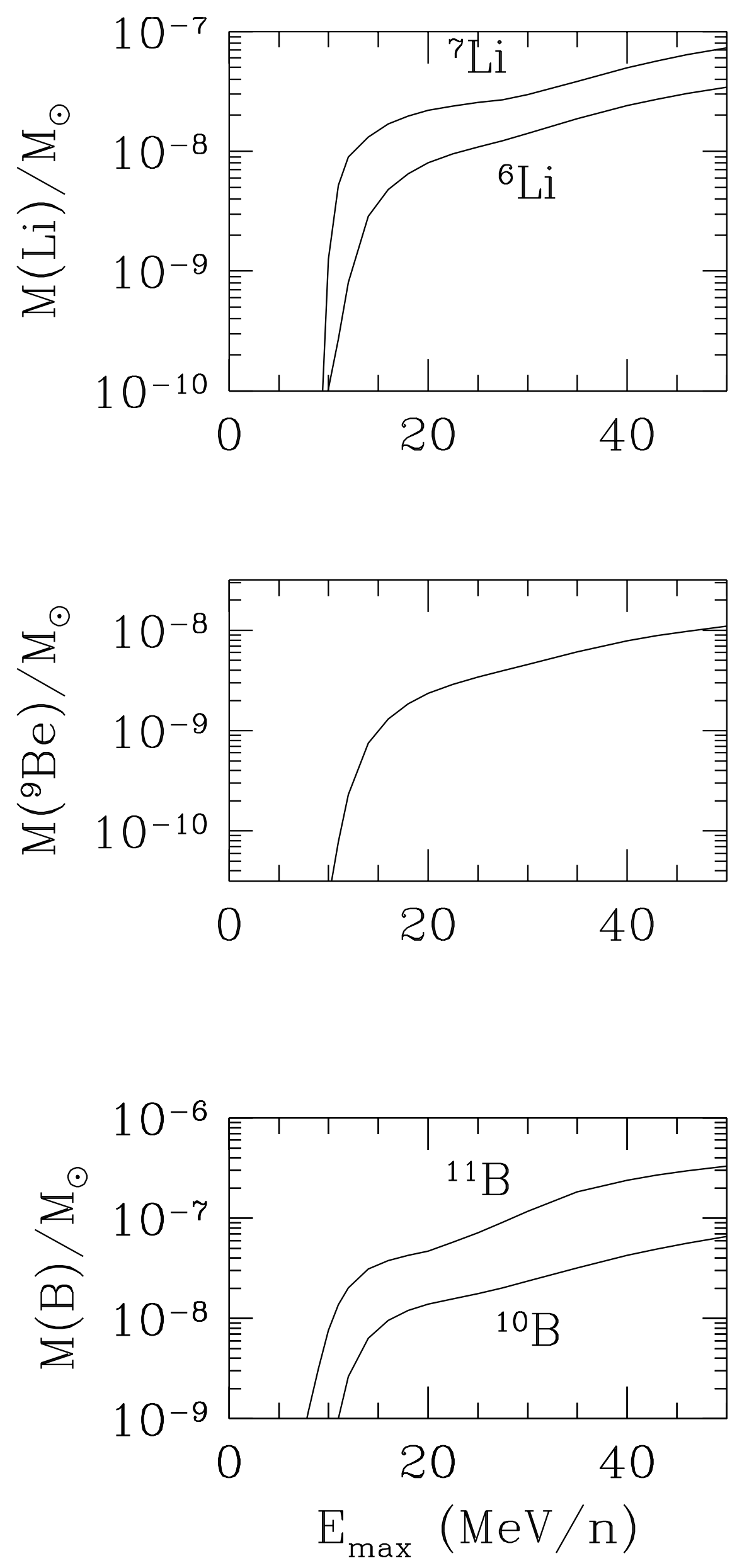

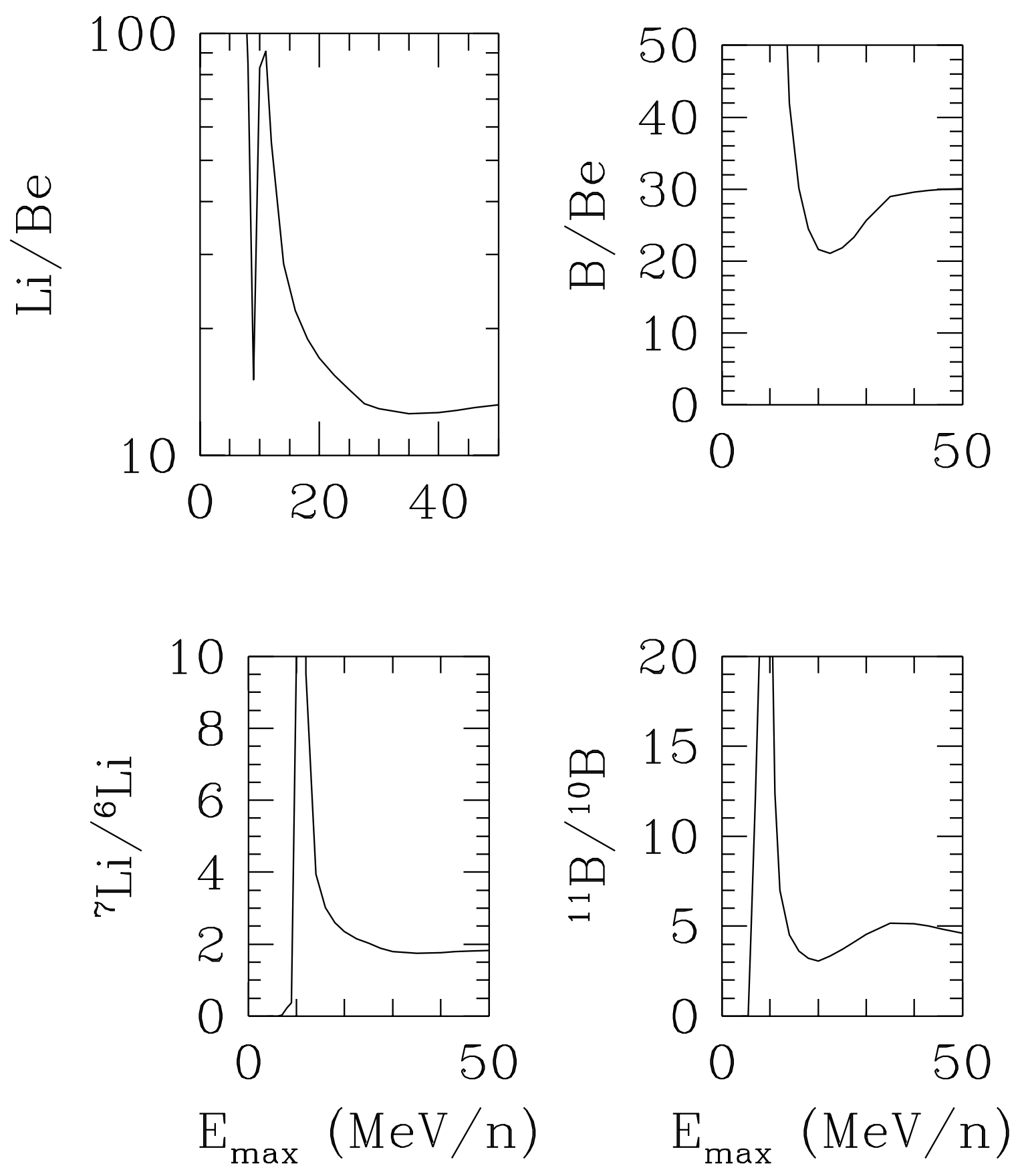